
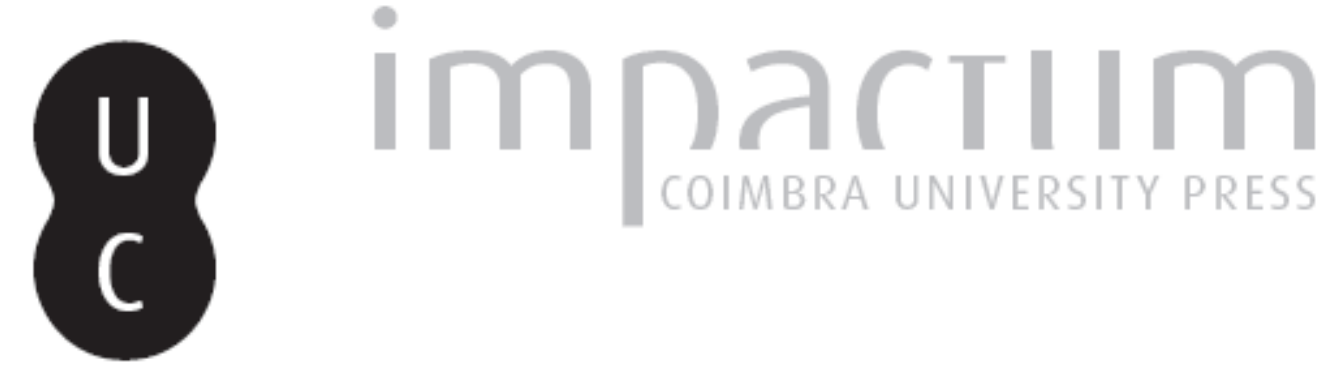

\title{
O artigo definido zero em dois contextos específicos no português do Brasil e no crioulo de Cabo Verde
}

Autor(es): $\quad$ Inverno, Liliana; Swolkien, Dominika

Publicado por: Faculdade de Letras da Universidade de Coimbra

URL persistente:

URI:http://hdl.handle.net/10316.2/38986

DOI:

DOI:http://dx.doi.org/10.14195/0870-4112_1_11

Accessed : $\quad$ 26-Apr-2023 10:57:34

A navegação consulta e descarregamento dos títulos inseridos nas Bibliotecas Digitais UC Digitalis, UC Pombalina e UC Impactum, pressupõem a aceitação plena e sem reservas dos Termos e Condições de Uso destas Bibliotecas Digitais, disponíveis em https://digitalis.uc.pt/pt-pt/termos.

Conforme exposto nos referidos Termos e Condições de Uso, o descarregamento de títulos de acesso restrito requer uma licença válida de autorização devendo o utilizador aceder ao(s) documento(s) a partir de um endereço de IP da instituição detentora da supramencionada licença.

Ao utilizador é apenas permitido o descarregamento para uso pessoal, pelo que o emprego do(s) título(s) descarregado(s) para outro fim, designadamente comercial, carece de autorização do respetivo autor ou editor da obra.

Na medida em que todas as obras da UC Digitalis se encontram protegidas pelo Código do Direito de Autor e Direitos Conexos e demais legislação aplicável, toda a cópia, parcial ou total, deste documento, nos casos em que é legalmente admitida, deverá conter ou fazer-se acompanhar por este aviso.

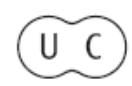




\section{Culturas em Diálogo}

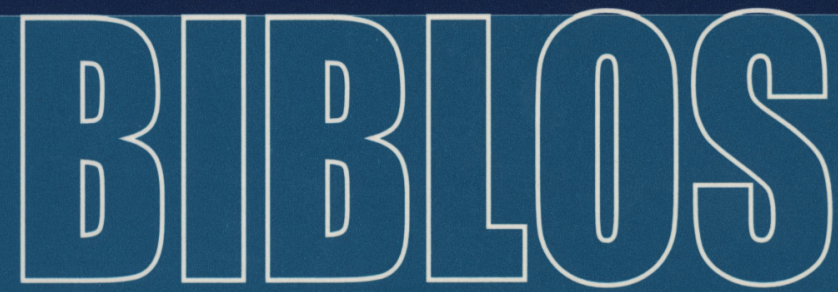




\section{$O$ Artigo definido Zero em Do is Contextos \\ Específicos no Português do Brasil \\ e no Crioulo de Cabo Verde 1}

\section{Introdução ${ }^{12}$}

Dizer que entre o Português do Brasil (PB) e o Português Europeu (PE) existem diferenças a todos os níveis linguísticos não constitui grande novidade, nem para linguistas, nem para os próprios falantes nativos do Português.

Os problemas começam quando se procuram avançar teorias explicativas para essas diferenças.

Até aos finais do século XVIII, não se punha a questão de distinguir o PB do PE. As próprias gramáticas da língua portuguesa não faziam qualquer distinção entre as duas variedades da língua. A ausência desta diferenciação é, aliás, visível nos próprios títulos publicados entre a descoberta do Brasil, em 1500, e os finais do século XVIII. Para apontar apenas alguns exemplos, dos muitos coligidos por Cardoso $^{3}$, Fernão de Oliveira intitulava a sua gramática do Português, datada de 1536, Grammatica da lingoagem portugueza e Pedro José de Figueiredo, publicando em 1799, intitulava a sua de Arte da Grammatica portugueza, ordenada em methodo breve, fácil e claro...

1 Este artigo resulta da comunicação apresentada na conferência conjunta da Associação Crioulos de Base Lexical Portuguesa e Espanhola e Society for Pidgin and Creole Linguistics, realizada em Coimbra, nos dias 28-29 de Junho de 2001.

$\wedge$ O presente trabalho não teria sido possível sem o incondicional apoio e orientação do Prof. Doutor John Holm e Doutora Ana Cristina Macário Lopes, pelo que deixamos aqui os nossos mais sinceros agradecimentos. Contudo, todos os erros e omissões são da nossa inteira responsabilidade.

${ }^{3}$ S. Cardoso, Historiografia gramatical (1500-1920): língua portuguesa - autores portugueses (Porto, Faculdade de Letras do Porto 1994). 
Só a partir do século XIX, principalmente após a Independência do Brasil em 1822, e o consequente desejo de afir mação de autonomia por parte dos brasileiros, reforçado pela impossibilidade de negar a existência de diferenças entre o PB e o PE, se iniciou o debate, que duraria até hoje, sobre as causas e a significação dessas divergências.

Até finais do século XIX, as dissemelhanças entre o PE e o PB seriam reconhecidas, principalmente ao nível do léxico, mas atribuídas à fala popular e justificadas, apenas, com base na evolução interna da língua portuguesa.

Porém, ainda no final desse século, Adolpho Coelho ${ }^{4}$, apontando semelhanças entre as características do PB e as características por ele observadas nos crioulos de base lexical portuguesa em África e na Ásia, veio explicar as diferenças entre o PB e o PE, com base no contacto do segundo com línguas indígenas e africanas que com ele coexistiram no espaço brasileiro. Esta explicação veio abrir caminho para uma série de abordagens africanistas e crioulistas extremamente produtivas na explicação das diferenças entre o PB e o PE e de que são exemplos os trabalhos de vários especialistas 5 .

Neste artigo, vamos analisar uma dessas diferenças, i.e., a omissão do artigo definido, no PB, em dois contextos específicos. Por um lado, enquanto determinante de sintagmas nominais (SN's) genericamente interpretados ( $\mathrm{f}$ $\mathrm{SN}(\mathrm{g})])$ e, por outro, em contexto imediatamente anterior à ocorrência dos adjectivos possessivos (1 Poss $\left.\left.\left(\mathrm{N}^{6}\right)\right]\right)$. No sentido de compreender as causas subjacentes a esta diferença entre PB e PE analisaremos, a seguir, o comportamento do artigo definido nos mesmos contextos no Crioulo de Cabo Verde (CCV).

4 F.A. Coelho, 'Os dialectos românicos ou neo-latinos na Africa, Asia e America', in J. Morais Barbosa (ed.), Estudos linguísticos crioulos (Lisboa, Academia Internacional de Cultura Portuguesa 1967) 2-234.

5 Vide J. Holm, 'Creole influence on Popular Brazilian Portuguese', in G. Gilbert (ed.), Pidgins and Creole Languages: essays in memory of John E. Reinecke (Honolulu, University Press of Hawaii 1987) 406-430; H. Mello, The genesis and development of Brazilian Vernacular Portuguese (Ann Arbor, University Microfilms International 1997); H. Couto, 'The question of (prior) creolization in Brazil', in M.Huber e M.Parkvall (editores), Spreading the word (London, University of Westminster Press 1999) 177-194; J. Holm, Languages in contact: the partial restructuring of vernaculars (Cambridge, Cambridge University Press no prelo).

^ 'Nome’ é neste artigo entendido como sinónimo de substantivo. 
$\mathrm{Na}$ senda da proposta de Macário Lopes', por 'SN's genericamente interpretados', entender-se-ão os SN's sujeitos de frases que predicam sobre um conjunto aberto de indivíduos susceptíveis de uma representação quantificacional. Essas frases admitem excepções ou contra exemplos, ou seja, não permitem generalizações e o intervalo de tempo que as valida não é temporalmente circunscrito, daí o uso de tempos como o presente do indicativo, o imperfeito e o futuro simples. Vejam-se os exemplos 'O gato é traiçoeiro' ou 'Os suecos comem carne de vaca'. 8

Tendo em conta a diversidade diatópica e diastrática inerente ao $\mathrm{PB}$, de tal forma acentuada que levou já à distinção no seio do mesmo de duas variedades - Português Vernáculo do Brasil (PVB) e Português Padrão do Brasil (PPB) - serão apresentados exemplos para cada uma destas variedades.

\section{O artigo definido no PE e no PB: dois usos distintos}

\section{1. [_ $\mathrm{SN}(\mathrm{g})]$}

Apesar de nenhuma língua apresentar SN's "distinctively generic in form" a verdade é que "many languages show a range of available noun phrase types with generic value, definite and indefinite, singular and non-singular"9. O português é uma dessas línguas e os SN's genericamente interpretados são introduzidos pelos artigos $o / a$, os/as e um/a. Vejam-se os exemplos:

PE: 1 'O castor constrói barragens'

PE: 2 'Os castores constroem barragens'

PE: 3 'Um castor constrói barragens'10

7 A.C. Macário Lopes, Aspectos da generacidade. Cadernos de Semântica. $n^{\circ} 6$ (Lisboa, Projecto JNICT-PCSH/LIN/212/91 1992).

8 A.C. Macário Lopes, Aspectos da generacidade. Cadernos de Semântica. $n^{\circ} 17$ (Lisboa, Projecto JNICT-PCSH/LIN/212/91 1993) 1.

9 Ch. Lyons, Definitness (Cambridge, Cambridge University Press 1999) 179.

10 A.C. Macário Lopes, Aspectos da generacidade. Cadernos de Semântica, n6 (Lisboa, Projecto JNICT-PCSH/LIN/212/91 1992) 10. 
No PB, nas suas variedades vernácula11 e padrão, o artigo definido neste contexto tende a não ocorrer, como se pode ver pelos exemplos seguintes:

PVB: 4 '0 cachorro come carne' 12

PPB: 5 \..0 galinha gosta muito de milho' ${ }^{12}$

No PE, neste contexto, a não ocorrência do artigo definido (singular ou plural) só é possível em frases proverbiais e aforísticas ou em títulos de textos de imprensa ${ }^{14}$, como no exemplo seguinte:

PE: 6 'Cão que ladra, não morde'

\subsection{I__ Poss (N)I}

Apesar de a tendência da língua portuguesa arcaica ser para a maior utilização da estrutura ' 0 Poss $(\mathrm{N}) \backslash$ actualmente, a norma europeia limita esta estrutura a fórmulas cristalizadas ('Vossa Excelência, Nosso Senhor') e substantivos em construções vocativas ('Não, minha senhora') ${ }^{15}$, sendo a não ocorrência do artigo definido nas restantes frases sentida como anómala.

PE: 7 'As nossas frequências são sempre difíceis'

* ‘0 Nossas frequências são sempre difíceis'

11 Segundo P. Teyssier, Manual da língua portuguesa (Portugal-Brasil) (Lisboa, Sá da Costa 1989) 105: "Na linguagem popular do Brasil, e na literatura que nela se inspira, suprime-se muitas vezes o artigo antes dos substantivos tomados num sentido geral e indeterminado, p.ex. 'Sempre me pareceu que mulher é um bicho esquisito".

12 E. Gartner, 'Particularidades morfo-sintácticas do português não padrão do Brasil, de Angola e de Moçambique'. Conferência apresentada na Faculdade de Letras da Universidade de Coimbra em 17.5.2000.

13 M.P. Sá et alii (editores), A linguagem falada culta na cidade do Recife: materiais para o seu estudo (Recife, Editora Universitária da UFPE 1996) 19.

14 A.C. Macário Lopes, Aspectos da generacidade. Cadernos de Semântica, n6 (Lisboa, Projecto JNICT-PCSH/LIN/212/91 1992) 10.

15 Ambos os exemplos citados por P. Teyssier, ob. cit., p. 135.

Faculdade de Letras | Universidade de Coimbra 
No PB, nas suas variedades vernácula e padrão, o artigo definido neste contexto tende a não ocorrer, como se pode ver pelos exemplos seguintes:

\author{
PVB: 8 'Quem sempre compra 0 nosso algodão é o Alfredo \\ Cesarino, 16 \\ PPB: 9 'ai 0 meu cachorro... 0 meu cachorro é lindo né? ${ }^{17}$
}

Da comparação dos exemplos do $\mathrm{PB}$ e do $\mathrm{PE}$ em ambos os contextos se conclui que no $\mathrm{PB}$, vernáculo e padrão, o artigo definido é tendencialmente omitido nos dois contextos em causa. Já no PE é obrigatório em ambos, salvo nos casos excepcionais supra-mencionados. Como explicar, então, esta discrepância? No sentido de dar resposta a esta questão, que se impõe depois da comparação feita e dos resultados obtidos, analisaremos o comportamento do artigo definido, nos mesmos contextos, no CCV.

\title{
2. I___ SN(g)| e I__ Poss (N)] no CCV
}

\subsection{Noções preliminares}

A escolha do Crioulo de Cabo Verde foi motivada pelo facto de, ao contrário de outros Crioulos Atlânticos de base lexical portuguesa (o Crioulo de São Tomé e Príncipe ou até o Crioulo da Guiné Bissau) o $\mathrm{CCV}$ ser aquele que mais se aproxima da língua portuguesa ${ }^{18}$, ou seja, de ser, entre os restantes, o menos reestruturado, sobretudo as variedades do Barlavento.

Ao escolher o CCV, estivemos também conscientes da grande diversidade linguística do mesmo. Diversidade esta que não se limita apenas à divisão dialectal entre as ilhas do Barlavento (Santo Antão, São Vicente, São Nicolau, Sal e Boavista) e as do Sotavento (Santiago, Fogo, Ática 1974)208.

16 A. Rodrigues, $O$ dialecto Caipira na região de Piracicaba (São Paulo, Editora

17 M.P. Sá et alii, ob.cit, p. 13

18 J. Morais-Barbosa, 'Cape Verde, Guinea-Bissau and São Tomé and Príncipe: the linguistic situation', in M. Valkhoff (ed.), Miscelânea Luso-Africana (Lisboa, Junta de Investigação Científica do Ultramar 1975) 137; J.Holm, Pidgins and Creoles (vol. 1 e II) (Cambridge, Cambridge University Press 1988) 272. 
Maio e Brava), pois, cada ilha apresenta, ainda, ao nível da fonética, da morfossintaxe e do léxico as suas particularidades específicas.

A heterogeneidade diatópica do CCV é ainda mais acentuada pela presença de um continuum crioulo resultante, essencialmente, de dois factores.

Por um lado, da existência de um contacto ininterrupto com a língua de superstrato ao longo dos 540 anos da colonização portuguesa. ${ }^{19}$ Este contacto, ainda que muitas vezes de baixa intensidade, devido ao número reduzido de europeus comparativamente ao número de africanos ${ }^{20}$ que permaneceram no arquipélago durante todo o processo de colonização, fez com que o nível de reestruturação do CCV seja menos profundo do que no caso de outros crioulos de base portuguesa (S. Tomé, Ano Bom).

Por outro lado, os ritmos e as épocas de colonização das ilhas do Barlavento e do Sotavento foram bem diferentes. Enquanto que, em 1582, a população adulta das ilhas de Santiago e Fogo chegava já aos 15000 , constituindo o grupo de europeus e livres $12,8 \%{ }^{21}$, o processo de colonização da ilha de São Vicente, a mais importante do ponto de vista linguístico no grupo de Barlavento foi começado só em 1794 e uma vez iniciado, o número de colonizadores europeus foi muito mais elevado do que nas ilhas do Sotavento ${ }^{22}$. Esta discrepância nas datas e nos padrões de colonização é um dos factores que permite explicar por que razão as ilhas do Sotavento apresentam um crioulo mais basilectal e as do Barlavento um crioulo mais acrolectal.

\footnotetext{
19 Note-se o paralelismo com a situação ocorrida no Brasil.
}

20 Mais uma vez, se nota aqui um paralelismo com a situação ocorrida no Brasil onde, de acordo com H. Mello, ob.cit, p. 85, "there was never a period in colonial and imperial Brazil when the white population was the majority. Indians were the majority in the first period (from 1538 to 1600), but they were replaced by the blacks and mulattos combined as the majority in all subsequent periods".

21 A. Bartens, 'A génese dos crioulos caboverdianos por difusão componencial e a importância dos estudos dialectológicosk in K. Zimmermann (ed.), Lenguas criollas de base lexical espanola y portuguesa (Madrid, Vervuert/Iberoamericana 1999) 68.

22 Vide D. Swolkien, 'Factores sociolinguísticos no povoamento da ilha de São Vicente de Cabo Verde' (Artigo apresentado na conferência anual da ACBLPE, A Coruna, 26-27 Junho de 2003, no prelo). 
Dado que faltam ainda estudos exaustivos e pormenorizados sobre a variedade linguística de cada ilha $^{23}$ e, especialmente, dado que não existem estudos comparativos relativos ao uso do artigo definido nas variedades do Barlavento e Sotavento, vamos tratar indistintamente ambas as variedades indicando, sempre que possível, a proveniência regional dos exemplos citados. Nas citações vai ser mantida a ortografia original.

\subsection{O artigo definido no CCV: um caso problemático}

A mera afirmação da existência do artigo definido no CCV $\{$ kel/kes - singular e plural, respectivamente) levanta objecções. Os primeiros trabalhos de Lopes da Silva, Almada e Veiga sobre o Crioulo de Cabo Verde ou constatam a inexistência do artigo definido no CCV, ou interpretam kel/kes apenas como formas demonstrativas ${ }^{24}$. É de salientar que os citados investigadores possuem a competência linguística de falantes nativos.

No entanto, há também, nas afirmações dos autores supra mencionados, muitas imprecisões ("O artigo definido pode dizer-se que não existe no Crioulo de Cabo Verde. A sua ausência foi remediada pelo uso do adjectivo demonstrativo aquele" ${ }^{25}$ ) e contradições. Assim, Veiga não menciona a existência da categoria do artigo definido, mas logo a seguir escreve "kel/kes ta sirbi pa adzetivu dimonstrativu, (pa artigu?) y pa pronómi dimostrativu”] ${ }^{26}$.

23 Há já, no entanto, algumas monografias [B. Lopes da Silva, $O$ dialecto Crioulo de Cabo Verde (Lisboa, Imprensa Nacional 1957); M. D. Almada. Cabo Verde contribuição para o estudo do dialecto falado no seu arquipélago (Lisboa, Junta de Investigação Científica do Ultramar 1961); E.A. Cardoso, O crioulo da ilha de S. Nicolau de Cabo Verde (Lisboa, Instituto de Cultura e Língua Portuguesa/Instituto Cabo-Verdiano do Livro 1989); D. Meintel, 'The Creole dialect of the island of Brava', in M. Valkhoff (ed ). Miscelânea Luso-Africana (Lisboa, Junta de Investigação Científica do Ultramar 1975) 205256] e estudos comparativos [(M.Veiga, Diskrison estrutural di Lingua Kabuverdianu (Lisboa, Institutu Kabuverdianu di Livru 1982) e, sobretudo, M. Baptista, The syntax of Cape Verdean Creole - Sotavento varieties (Amsterdam/ Philadelphia, John Benjamins 2002)]. ob. cit. p.77.

24 Vide B. Lopes da Silva, ob. cit, p. 129; M.D. Almada, ob. cit. p.89; M. Veiga.

25 M.D. Almada, ob. cit. p. 89.

26 M. Veiga, ob. cit. p. 87. Para um resumo e crítica das obras sobre CCV - desde 1886 até o ano 1994 - que tocam na questão do sistema de artigos veja-se C. Albino, Para o estudo do Crioulo falado pela comunidade Cabo-Verdiana radicada em Portugal variação e mudança no sistema de artigos (Lisboa, Faculdade de Letras da Universidade de Lisboa 1994). 
As investigações mais recentes de Lucchesi ${ }^{27}$ e as publicações de Baptista ${ }^{28}$ surgem como resposta às propostas de Bickerton e Janson ${ }^{29}$ e reconhecem claramente a existência da categoria do artigo definido no CCV.

Há, no entanto, algumas divergências na posição de Lucchesi e Baptista. Lucchesi considera que a presença ou ausência do artigo definido no CCV resulta apenas de uma variação estilística ("From this irregularity in the use of the definite article, it could be inferred that its use would be related to discourse and/or stylistic factors") ${ }^{30}$. No entanto, Baptista diverge da opinião de Lucchesi e postula que kel/kes fazem parte de um complexo sistema referencial do CCV, complexidade essa que reside na maneira como "overt determiners interact with null articles in marking specifity and nonspecifity of NPs"31.

O trabalho de Albino - a análise de um amplo material empírico recolhido entre os emigrantes cabo-verdianos radicados na zona da Grande Lisboa, cujos resultados foram comparados com os textos recolhidos por Parsons ${ }^{32}$ na segunda década do século $\mathrm{XX}$, aponta, por seu lado, para uma mudança relativamente a um maior uso da marca kel como artigo definido para marcar os SNs específicos pressupostos ${ }^{33}$. Apesar dessa mudança, a marca zero continua a ser uma marca de definição por excelência no $\mathrm{CCV}^{34}$, particularmente nos contextos em causa nesta apresentação.

27

D. Lucchesi, The article system of Cape Verde and São Tomé Creole Portuguese: general principles and specific factors'. Journal of Pidgin and Creole Languages 8:1 (1993) 81-108.

28 M. Baptista, The morpho-syntax of nominal and verbal categories in Capeverdean Creole (Ann Arbor, University Microfilms International 1997) e ob. cit.

29 Sobre as propostas de Bickerton e Janson veja-se D. Lucchesi , ob.cit. pp. 8184.

30 D. Lucchesi, ob. cit. p.94.

31 M. Baptista, The morpho-syntax of nominal and verbal categories in Capeverdean Creole (Ann Arbor, University Microfilms International 1997) 10, 24-31.

32 E. Parsons, Ten Folk-Tales from the Cape Verde Islands', Journal of American Folk-Lore 30 (1917) 230-238.

33 C. Albino, ob. cit. pp. 164-165. Se este aumento resulta de uma possível descrioulização, ou seja, de um movimento na direç̧ão do português provocado pelo contacto constante com a língua de superstrato, será já outra questão.

34 M. Baptista, The syntax of Cape Verdean Creole - Sotavento varieties (Amsterdam/ Philadelphia, John Benjamins 2002) 34.

Faculdade de Letras I Universidade de Coimbra 


\title{
2.3. I__ $\mathrm{SN}(\mathrm{g}) \mid$ no $\mathrm{CCV}$
}

No CCV os SNs genericamente interpretados são sistematicamente introduzidos pela marca zero ${ }^{35}$.

\author{
CCV: 10 '0 Katchupa ta dura tres ora pa sta prontu’36 (Santiago) \\ ${ }^{4}$ A catchupa leva três horas a ficar pronta' \\ CCV: 11 "0 Avestrus ten àza má'l ka ta bwa'-'7 (São Nicolau) \\ 'A avestruz tem asas. mas não voa'
}

\subsection{I__ Poss (N)| no CCV}

Também neste contexto o artigo definido no CCV não ocorre. Segundo Lucchesi "definite article in Cape Verdean and São Tomense is absent with other modifiers-possessives etc." 38 .

\author{
CCV: 12 'Lob tra 0 se casac' ${ }^{39}$ (Fogo) \\ 'O Lobo tirou o seu casaco'
}

CCV: 13 '...0 nha kamiza de note'40 (Santiago)

l.. a minha camisa de noite'

\section{3. [_ $\mathrm{SN}(\mathrm{g}) \mid$ e I__ Poss (N)] no CCV e PB: uma comparação}

Pela análise dos exemplos do CCV nos contextos em causa, conclui-se que o artigo definido não ocorre em nenhum. Este comportamento é semelhante ao comportamento do artigo definido analisado no PB, no qual, nas suas variedades vernácula e padrão, o artigo

33 C. Albino, ob. cit. 117.

3^ D. Fanha, [cf. D.Pereira], 'Aspectos do contacto entre o Português e o Crioulo de Cabo Verde'. Separata do Congresso sobre a situação actua! de Língua Portuguesa no mundo (Lisboa, Instituto de Cultura e Língua Portuguesa 1987) 301.

3^ E.A. Cardoso, ob. cit. p. 107.

38 D. Lucchesi, ob. cit. p.91. "É de facto muito pouco provável (no CCV) uma construção desse tipo det+poss+N. Talvez só num texto mais ou menos literário, ou em falantes muito permeáveis à norma portuguesa. A presença do possessivo "anula" a presença de uma marca de definição, não há necessidade de ser redundante". (Albino, comunicação via e-mail, 23 Maio 2001).

${ }^{3 \wedge}$ E. Parsons, ob. cit. p. 230.

C. Albino, ob. cit. p. 127. 
definido é tendencialmente omitido, por oposição à exigência do mesmo pelo PE. O paralelismo entre o CCV e o PB é, portanto, evidente:

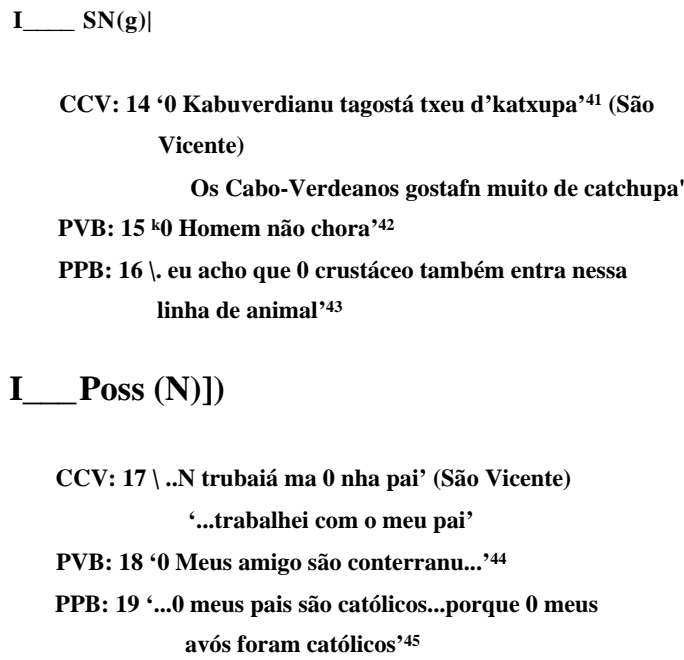

\section{A divergência no PB e no PE e o paralelismo no PB e CCV: algumas hipóteses explicativas}

Como explicar, então, a existência desta discrepância no uso europeu e brasileiro do artigo definido nos contextos em causa, por um lado, e o paralelismo no uso do mesmo no PB e no CCV?

Sem procurar apresentar justificações definitivas, parece-nos, com base na análise dos exemplos acima apresentados, que a resposta a esta questão reside, numa explicação externa para $\mathrm{SN}(\mathrm{g})]$ e numa explicação interna para [__ Poss $(\mathrm{N})]$.

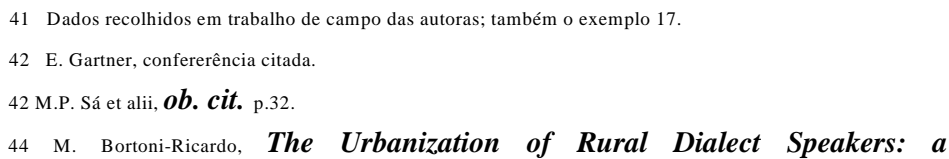
sociolinguistic study in Brazil (Cambridge, Cambridge University Press 1985) 128.

42 M.P. Sá et alii, ob. cit. p. 71. 


\section{1. $\left[\_\right.$_ $\mathrm{SN}(\mathrm{g}) \mid$ Explicação externa}

Neste contexto, a não ocorrência do artigo definido, tanto no $\mathrm{PB}$ como no $\mathrm{CCV}$, terá sido motivada pelo contacto da língua de superstrato com línguas de substrato desprovidas de artigo definido do grande grupo Niger-Congo ${ }^{46}$;Yoruba e Banto, entre outras, no caso do $\mathrm{PB}$ e Mandinga e Wolof, entre outras, no caso do $\mathrm{CCV}^{47}$. Escravos Mandinga e Wolof chegavam também ao Brasil vindos do entreposto de Cabo Verde, ou directamente da Guiné ${ }^{48}$. No caso do PB há ainda a acrescentar o contacto do português com variedades já reestruturadas do mesmo levadas para o Brasil pelos escravos e o contacto com as línguas da família Tupi-Guarani faladas pelos índios brasileiros. Nestas, segundo Navarro, não existiam artigos de qualquer espécie.

Tupi: 20 “Os índios ficam na enseada do rio” fAbá o-pytá "y

kúá-pe’)

'Uns índios ficam na enseada' ('Abá o-pytá “y kíiá-pe’)

Em Tupi as duas frases ficam iguais. É o contexto que, geralmente, nos esclarece o sentido exacto ${ }^{49}$.

Aparentemente, a marca 0 para a definição, neste contexto, seria típica das línguas reestruturadas. Holm afirma, a este propósito, que "the creoles surveyed all use bare nouns to convey generic meaning" e considera válida a proposta de Bickerton, segundo a qual, "the Atlantic

46 Nas línguas desta família a determinação está a cargo de diversos marcadores que em nada se identificam com o artigo definido português. A atestá-lo estão as obras de P.O. Ogunbowale, The Essentials of the Yoruba Language (London, University of London Press 1977); D. Creissels, Eléments de grammaire de la langue Mandinka (Grenoble. Publications de l'Université des Langues et Lettres 1983); S. Sauvageot, Description synchronique d'un dialecte Wolof: le parler du Dyolof (Dakar 1965) e Ch. Bird. J. Hutchinson e M. Kanté, An Ka Bamanankan Kalan: Beginning Bambara (Bloomington. Indiana University Linguistics Club 1976-1977) nas quais a categoria artigo definido não é mencionada.

47 Mandinga é responsável por $60 \%$ do léxico africano no CCV enquanto Wolof é responsável por $20 \%$, segundo J.L. Rougé, 'Apontamentos sobre o léxico de origem africana dos crioulos da Guiné e de Cabo Verde (Santiago), in K. Zimmermann (ed.), ob. cit. 60.

48 "During at least the first and second waves of slave imports, the ports of Cape Verde, Guinea and São Tomé in West Africa were continuously used as entrepôts for the slave trade" (H. Mello, ob. cit. p. 55).

Vozes 1998) 7.

49 E. Navarro, Moderno do Tupi Antigo: a lingua do Brasil (Petrópolis, Editora Faculdade de Letras Universidade de Coimbra 
creoles use a singular noun without an article to refer to general categories unlike their lexical source languages." 50

Para a explicação da não ocorrência do artigo definido neste contexto não será, ainda, de excluir o facto de as formas do mesmo em português (o/a, os/as) não terem a estrutura silábica $\mathrm{CV}$ das línguas africanas, serem átonas e por isso facilmente amalgamáveis ou omitidas $^{51}$. Há vestígios dessa amálgama na toponímia do Arquipélago de Cabo Verde: Skórde (os Cardos) e Spédrã (as Pedras) e no léxico: stóxa (as tochas) e ãzílã (as ilhas) ${ }^{52}$. Também no PVB encontramos exemplos representativos do mesmo fenómeno: 'zóyo' ('os olhos'). ${ }^{53}$.

A não ocorrência do artigo definido neste contexto no PB e no CCV vai, de resto, não só contra a norma do PE, mas também contra a norma de outras línguas românicas, como o francês e o espanhol, nas quais os SNs genericamente interpretados são sempre introduzidos por artigo definido ou indefinido em regime de distribuição complementará 4

Francês: 'Le/Un castor construit des barrages.' 55

Espanhol: 'El/Un castor construye embalses'

\section{2. [__ Poss (}

Neste contexto a não ocorrência do artigo definido, no PB e no $\mathrm{CCV}$, pode ser explicada através do próprio desenvolvimento diacrónico da língua portuguesa. No PE dos séculos XV e XVI (português moderno em formação), quando os primeiros colonizadores começaram a chegar, tanto ao Brasil como a Cabo Verde, existia uma clara tendência para a não utilização do artigo definido neste contexto, por oposição à norma actual que requer a anteposição do artigo ao adjectivo possessivo.

\section{${ }^{5 \wedge}$ J. Holm, An introduction to Pidgins and Creoles (Cambridge, Cambridge University Press 2000).}

51 Da mesma opinião é E. W. Thomas, The Syntax of Spoken Brazilian Portuguese (Nashville, Vanderbilt University Press 1969) 17 para quern "'the fact that the singular articles consist of a single vowel, while the plural forms begin with a vowel, is of considerable importance. When these vowels come into contact with like vowels in speech, the two tend to fuse into one, so that there is no longer an audible, separate vowel of the article... When the article is no longer audible, it tends to be lost as a necessary part of the sentence."

52 B. Lopes da Silva, oh. cit. p.129.

53 H. Mello, ob. cit.p. 224.

$O$ artigo indefinido nâo pode, porém, surgir com SNs genericamente interpretados inseridos em frases cujo predicado denote propriedades específicas da espécie.

55 Ch. Lyons, ob. cit. p. 185.

Faculdade de Letras | Universidade de Coimbra 
Segundo Said Ali, "Não podemos precisar a época do aparecimento desta segunda forma... [i.e. $\longrightarrow$ Poss $(\mathrm{N})$ ] ... certo é que o seu emprego era relativamente restrito e só de Camões para cá se torna, de século para século, cada vez mais notória... ." 56 Para o mesmo tipo de explicação interna apontam também Narro e Scherre ${ }^{57}$.

Além disso, do ponto de vista da pragmática da comunicação, achamos que a dupla determinação do nome, por artigo e adjectivo possessivo, é uma construção redundante. Esta redundância era reconhecida pelos falantes do português arcaico, razão pela qual o artigo era maioritariamente omitido em posição anterior à ocorrência de adjectivo possessivo. A utilização do artigo neste contexto constituía uma construção marcada, reservada apenas a algumas intenções pragmáticas específicas, nomeadamente, quando o falante pretendia exprimir afectividade ou colocar uma maior ênfase no 'adjectivo possessivo' ou grupo 'adjectivo possessivo + nome.'

$\mathrm{O}$ PE, seguindo a tendência inovadora de outras línguas românicas, como por exemplo, o romeno, o catalão e o italiano, generalizou o uso desta dupla determinação, enquanto o PB, tal como o francês e o espanhol, a abandonou. ${ }^{58}$ Em nossa opinião, a opção pela não ocorrência do artigo definido em [___ Poss (N)] no PB poderá ter sido, mais uma vez, influenciada pela sintaxe das línguas da família Tupi. Segundo Navarro "em rigor, em Tupi não existem pronomes possessivos nem adjectivos possessivos. Os possessivos são, na verdade, pronomes pessoais em relação genitiva (que se obtém, em Tupi, invertendo-se o nome da coisa possuída e o nome do possuidor p.ex. faca de Pindobuçu: Pindobusu kysé)" 59 . Uma explicação externa para a não ocorrência do artigo definido neste contexto não será, portanto, de excluir.

M. Said Ali, Gramática Histórica da Língua Portuguesa (Sào Paulo. Melhoramentos 1966) 96.

57 A. Narro \& M. Pereira Scherre, 'Variable concord in Portuguese: the situation in Brazil and Portugal', in J. McWhorter (ed.), Language Change and Language Contact in Pidgins and Creoles (Amsterdam, Philadelphia, John Benjamins 2000) 235-255.

58 R. Lapesa, Estúdios de Morfosintaxis Histórica del Espahol (Madrid. Credos 2000) 433, sumaria esta questão da seguinte forma: 'Las lenguas românicas se enfrentaron desde sus origenes con la concurrencia entre el posesivo aplicado al nombre sin otra compafía y el posesivo precedido de artículo.... la concurrencia procedia de una oposición de intensidad expresiva: el posesivo nudo era el término no mareado frente al posesivo con artículo, que destacaba al sustantivo y su relación con el poseedor.'

59 E. Navarro, ob. cit. p.33. 


\section{Conclusões}

Em suma, entre o PB e o PE existem diferenças significativas a todos os níveis linguísticos, significativas ao ponto de tanto Teyssier como Cunha e Cintra sentirem necessidade de, nas suas gramáticas, chamar a atenção para a existência de duas normas do português ${ }^{60}$.

Procurou demonstrar-se neste artigo que as causas dessas diferenças podem e devem ser explicadas por processos de mudança da língua portuguesa motivados por condicionalismos tanto internos como externos. A origem de cada diferença estrutural deve ser estudada caso a caso, pesando cuidadosamente os argumentos a favor de um ou outro condicionalismo, ou a favor da convergência de ambos.

60 P. Teyssier, ob. cit. p. 15 e Cunha e Cintra pp. 13-14.

Faculdade de Letras Universidade de Coimbra 